\title{
BMJ Open Is high-sensitivity troponin, alone or in combination with copeptin, sensitive enough for ruling out NSTEMI in very early presenters at admission? A post hoc analysis performed in emergency departments
}

Camille Chenevier-Gobeaux, ${ }^{1}$ Mustapha Sebbane ${ }^{2}$ Christophe Meune, ${ }^{3,4}$ Sophie Lefebvre, ${ }^{2}$ Anne-Marie Dupuy, ${ }^{5}$ Guillaume Lefèvre, ${ }^{6,7}$ Nicolas Peschanski, ${ }^{8}$ Patrick Ray ${ }^{9,10}$

To cite: Chenevier-Gobeaux C, Sebbane M, Meune C, et al. Is high-sensitivity troponin, alone or in combination with copeptin, sensitive enough for ruling out NSTEMI in very early presenters at admission? A post hoc analysis performed in emergency departments. BMJ Open 2019;9:e023994. doi:10.1136/ bmjopen-2018-023994

- Prepublication history for this paper is available online. To view these files, please visit the journal online (http://dx.doi. org/10.1136/bmjopen-2018023994).

Received 12 June 2018 Revised 11 March 2019 Accepted 12 March 2019

A) Check for updates

(C) Author(s) (or their employer(s)) 2019. Re-use permitted under CC BY-NC. No commercial re-use. See rights and permissions. Published by BMJ.

For numbered affiliations see end of article.

Correspondence to Dr Camille Chenevier-Gobeaux; camille.gobeaux@aphp.fr

\section{ABSTRACT}

Objectives Copeptin and high-sensitivity cardiac troponin (HS-cTn) assays improve the early detection of nonST-segment elevation myocardial infarction (NSTEMI). Their sensitivities may, however, be reduced in very early presenters.

Setting We performed a post hoc analysis of three prospective studies that included patients who presented to the emergency department for chest pain onset (CPO) of less than 6 hours.

Participants 449 patients were included, in whom $12 \%$ had NSTEMI. CPO occurred $<2$ hours from ED presentation in 160, between 2 and 4 hours in 143 and $>4$ hours in 146 patients. The prevalence of NSTEMI was similar in all groups $(9 \%, 13 \%$ and $12 \%$, respectively, $p=0.281)$.

Measures Diagnostic performances of HS-cTn and copeptin at presentation were examined according to CPO. The discharge diagnosis was adjudicated by two experts, including cardiac troponin I (cTnl). HS-cTn and copeptin were blindly measured.

Results Diagnostic accuracies of cTnl, cTnl +copeptin and HS-cardiac troponin T (HS-cTnT) (but not HS-cTnT +copeptin) lower through CPO categories. For patients with $\mathrm{CPO}<2$ hours, the choice of a threshold value of $14 \mathrm{ng} / \mathrm{L}$ for HS-cTnT resulted in three false negative (Sensitivity 80\%(95\% Cl 51\% to 95\%); specificity 85\% (95\% Cl $78 \%$ to $90 \%$ ); $79 \%$ of correctly ruled out patients) and that of $5 \mathrm{ng} / \mathrm{L}$ in two false negative (sensitivity $87 \%$ (95\% Cl $59 \%$ to $98 \%$ ); specificity $58 \%$ (95\% Cl $50 \%$ to $66 \%) ; 52 \%$ of correctly ruled out patients). The addition of copeptin to HS-cTnT induced a decrease of misclassified patients to 1 in patients with CPO $<2$ hours (sensitivity $93 \%$ (95\% Cl $66 \%$ to $100 \%)$; specificity $41 \%$ (95\% Cl $33 \%$ to $50 \%)$ ).

Conclusion A single measurement of HS-cTn, alone or in combination with copeptin at admission, seems not safe enough for ruling out NSTEMI in very early presenters (with CPO $<2$ hours).

Trial registration number DC-2009-1052
Strengths and limitations of this study

Focus on very early chest pain presenters that was not performed before.

- Small numbers of very early chest pain presenters, although the data grouping of three previous studies.

- A single measurement of troponin at admission was considered for the analysis, but not its kinetics.

- The gold-standard diagnosis was based on a non-high-sensitivity cardiac troponin.

\section{INTRODUCTION}

The management of patients with acute chest pain at the emergency department (ED) is a major health problem and an adequate ruling out process for non-ST segment elevation myocardial infarction (NSTEMI) is crucial. Cardiac troponin (cTn) measurement with conventional assays and more recent high-sensitivity cTn assays (HS-cTn, either isoforms HS-cTnI or HScTnT) are current diagnostic tools for the assessment of these patients. ${ }^{1}$ The European Society of Cardiology (ESC) guidelines proposed a rapid rule out strategy using low HS-cTn values (ie, values below the limit of detection of the assay (LoD)) as decisional threshold for ruling out NSTEMI. ${ }^{1}$ The accuracy of a rapid 0/1-hour algorithm has also been recently demonstrated in patients with chest pain onset $(\mathrm{CPO})<6$ hours $^{2}$ and is endorsed by the latest ESC guidelines. ${ }^{1}$ In this analysis, the authors indicated that using a single HS-cardiac troponin $\mathrm{T}$ (HS-cTnT) cut-off value of $14 \mathrm{ng} / \mathrm{L}$ at presentation resulted in $88.7 \%$ sensitivity and $97.3 \%$ negative predictive value 
(NPV), but that this single assay strategy was less effective than the combination of absolute level of HS-cTnT measured at presentation and again at 1 hour, combined with the absolute difference between the two levels. ${ }^{2}$

However, in very early presenters, such rapid and efficient triage at presentation may be uncertain. ${ }^{3}$ Indeed, very early presenters, defined as having chest pain $<2$ hours, are considered by some authors as highly vulnerable as they may not present all symptoms and signs and thus be exposed to a higher risk of misdiagnosis and therefore worse outcome ${ }^{4}$; the application of a rapid algorithm to these patients may lead to early discharge within 3 hours from ED admission. Recently, a meta-analysis (based on 11 studies) indicated that a single HS-cTnT concentration below the LoD may successfully rule out acute MI (AMI). ${ }^{5}$ However, concerns have been raised about the safety of a single measurement rule out protocol performed at presentation in early presenters. ${ }^{67}$

Copeptin (in association with cTn) assays improve the early detection of NSTEMI. ${ }^{8-12}$ Indeed, a randomised controlled trial demonstrated the safety of early discharge using a single combination of copeptin $+\mathrm{cTn}$ at presentation for patients with $\mathrm{CPO}<6$ hours. ${ }^{13}$

The present study aimed to assess the diagnostic performance of HS-cTn and copeptin as a single measurement at admission in very early ED presenters with suspected NSTEMI.

\section{PATIENTS AND METHODS}

\section{Study design and population}

The study is a post hoc analysis of three French prospective clinical studies (already published) of cardiac biomarker testing, each to explore the usefulness of copeptin and/or HS-cTnT testing in patients who presented to the ED with acute chest pain of less than 6 hours. ${ }^{14}{ }^{15}$ However, none of these studies evaluated the influence of CPO value on diagnostic performances. All three trials had comparable inclusion/exclusion criteria and gathered similar clinical information (table 1). Patients requiring renal replacement therapy were excluded. Recommendations of the Standards for Reporting of Diagnostic Accuracy initiative were applied. ${ }^{16}$

\section{Patient involvement}

The development of the research question was not informed by patients. Patients were not involved in the design of this study. Patients were not involved in the recruitment to and conduct of the study. There was no results dissemination to study participants.

\section{Routine assessment}

All patients underwent an initial clinical evaluation (including clinical history, physical examination, 12-lead ECG, pulse oximetry, routine blood tests and chest X-ray). Conventional cardiac troponin I (cTnI) was measured on a venous blood collection performed at presentation and, if required, repeated after 3-9 hours, as clinically indicated. ${ }^{17}$ The CPO, defined as the delay from symptom onset to presentation, was recorded, based on patient history/declaration. When history was incomplete or inconsistent, CPO was not recorded and patient was excluded from the analysis (see flow chart, figure 1). Based on all clinical, biological (including cTnI value, but not HS-cTnT and copeptin values which were blindly measured) and imaging results, a decision was made by the attending physician to admit or discharge the patient, as well as medical therapy and revascularisation if indicated. Attending emergency physicians and cardiologists were blinded to the results of HS-cTnT and copeptin, and biologists were blinded to the suspected diagnosis at presentation.

Patients with no cTnI results and/or no recorded CPO value and patients with a final diagnosis of STEMI were excluded (see flow chart, figure 1).

\section{Gold-standard diagnosis}

The gold-standard diagnosis was adjudicated by two independent experts (emergency physician and cardiologist)

Table 1 Main characteristics of original study designs

\begin{tabular}{|c|c|c|}
\hline & Sebbane et $a l^{14}$ & Chenevier-Gobeaux et al $^{15}$ \\
\hline Exclusion criteria & $\begin{array}{l}\text { Patients with traumatic causes of chest } \\
\text { pain. }\end{array}$ & $\begin{array}{l}\text { Patients }<18 \text { years old. } \\
\text { Acute or chronic renal failure requiring dialysis. }\end{array}$ \\
\hline $\begin{array}{l}\text { Plasma sampling and } \\
\text { storage }\end{array}$ & $\begin{array}{l}\text { Heparinised and } \\
\text { ethylenediaminetetraacetic acid blood } \\
\text { collection. Storage at }-80^{\circ} \mathrm{C} \text { for later } \\
\text { analysis. }\end{array}$ & $\begin{array}{l}\text { Heparinised blood collection after routine cardiac troponin I } \\
\text { measurement. Storage at }-40^{\circ} \mathrm{C} \text { until HS-cTnT and copeptin } \\
\text { measurement. }\end{array}$ \\
\hline Registration no/name & $\begin{array}{l}\text { French Health Ministry (no. DC-2009- } \\
\text { 1052). }\end{array}$ & $\begin{array}{l}\text { French Local Ethic comity « Comité de Protection des } \\
\text { Personnes Ile-de-France » III (Hôpital Cochin) et VI (Centre } \\
\text { Hospitalier Universitaire Pitié-Salpétrière). }\end{array}$ \\
\hline
\end{tabular}



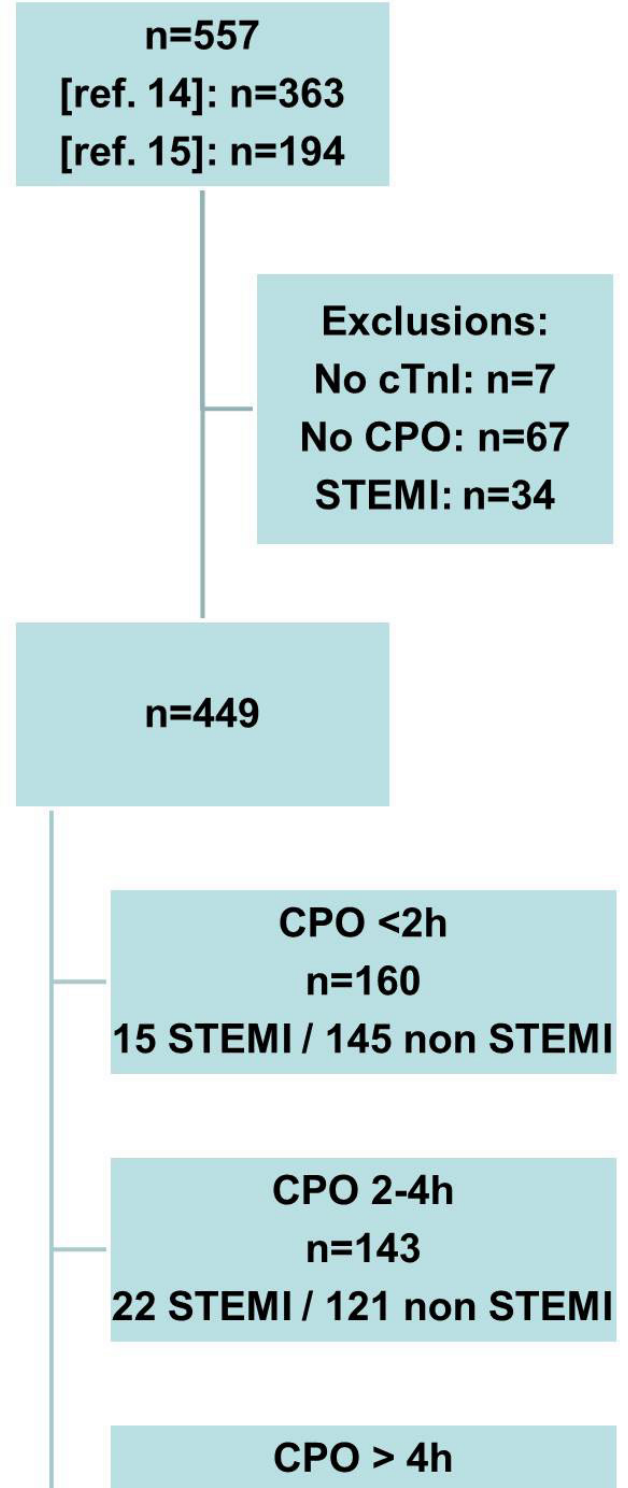

$n=146$

18 STEMI / 128 non STEMI

Figure 1 Flow chart of the studied population. CPO, chest pain onset; cTnl, cardiac troponin I; STEMI, ST-segment elevation myocardial infarction.

who reviewed all available medical records (including patient history, physical findings, laboratory results including cTnI value and radiological testing, ECG, echocardiography, cardiac exercise test, coronary angiography, summary chart at discharge) pertaining to the patient from the time of ED presentation to 30-day follow-up. Experts were blind to copeptin and HS-cTnT results. In the event of diagnostic disagreement, cases were reviewed and adjudicated in conjunction with a third expert.

AMI was diagnosed according to the universal definition that was in force at the time of inclusions and adapted to the use of a conventional cTn. ${ }^{18}$ Thus, patients with a cTnI increase (or a rise/fall pattern) above the $10 \%$ coefficients of variation (CV) threshold, associated with at least one of the following: symptoms of myocardial ischaemia, new ST-T changes or new $Q$ wave on ECG, imaging of new loss of viable myocardium or normal cTnI on admission were classified as having an MI (STEMI with an ST elevation in at least two continuous leads on ECG or new onset of left bundle branch block or NSTEMI). Patients with STEMI were excluded from the analysis, based on ST elevation observed on the ECG (see flow chart, figure 1). Patients were classified according to the CPO, $<2$ hours, from 2 to 4 hours and $>4$ hours; those with a $\mathrm{CPO}<2$ hours were considered as very early presenters.

Unstable angina (UA) diagnosis was adjudicated in patients with history or clinical symptoms consistent with acute coronary syndrom but without ST-T wave changes on the ECG and without change of cTn on serial testing. Other diagnostic categories besides NSTEMI and UA were non-ACS (eg, stable angina, myocarditis, arrhythmias, heart failure, pulmonary embolism and chest pain of unknown origin). UA and non-ACS chest pain were considered as non-NSTEMI in our analysis.

\section{Troponin measurements}

Plasma cTnI concentrations were routinely measured on an X-pand HM analyzer, using the cTnI immunoassay (Siemens Healthcare Diagnostics, Newark, Connecticut , USA) in two EDs (Cochin and La Pitié Salpêtrière Hospitals). The LoD was $0.04 \mu \mathrm{g} / \mathrm{L}(40 \mathrm{ng} / \mathrm{L})$. The limit of quantitation (LoQ), that is, the $10 \%$ imprecision point (or $10 \% \mathrm{CV}$ ), which is the lowest cTn concentration that can be reproducibly measured with a between-run CV of $\leq 10 \%$, was $0.14 \mu \mathrm{g} / \mathrm{L}(140 \mathrm{ng} / \mathrm{L})$. The 99 th percentile of the assay was $0.07 \mu \mathrm{g} / \mathrm{L}(70 \mathrm{ng} / \mathrm{L})$, with CVs between $15 \%$ and $22 \%$. The measuring range was $0.04-40 \mu \mathrm{g} / \mathrm{L}$ (40-40000 ng/L), and the imprecision values across the measuring range were below $10 \%$.

In Bicêtre and in Montpellier hospitals, plasma cTnI concentrations were routinely measured on an Access analyser (Beckman Coulter, Brea, California, USA). According to the manufacturer's data, the LoD was $0.01 \mu \mathrm{g} / \mathrm{L}(10 \mathrm{ng} / \mathrm{L})$, the $20 \%$ point on the imprecision curve was $0.02 \mu \mathrm{g} / \mathrm{L}(20 \mathrm{ng} / \mathrm{L})$. The $\mathrm{LoQ} / 10 \% \mathrm{CV}$ was $0.04 \mu \mathrm{g} / \mathrm{L}(40 \mathrm{ng} / \mathrm{L})$. The 99 th percentile of the assay was $0.04 \mu \mathrm{g} / \mathrm{L}(40 \mathrm{ng} / \mathrm{L})$. The measuring range was $0.01-$ $100 \mu \mathrm{g} / \mathrm{L}(10-100000 \mathrm{ng} / \mathrm{L})$, and the imprecision values across the measuring range were below $10 \%$.

After routine cTnI measurement, plasma samples were aliquoted and frozen $\left(-40^{\circ} \mathrm{C}\right)$ until HS-cTnT and copeptin measurement.

Hs-cTnT was measured in heparinised collected samples, on an Elecsys2010 analyser (Roche Diagnostics, Meylan, France). The limit of blank (LoB) was $3 \mathrm{ng} / \mathrm{L}$, the LoD was $5 \mathrm{ng} / \mathrm{L}$ and the 99 th percentile was $14 \mathrm{ng} / \mathrm{L}$. The measuring range was 3-10000 ng/L. In our laboratory, CVs obtained in Roche quality controls containing 27.5 and $2360 \mathrm{ng} / \mathrm{L}$ of cTnT were $3.6 \%$ and $2.8 \%$ (between-run precision) and $1.4 \%$ and $0.4 \%$ (within-run precision). Of note, the LoD is measured with a between-run CV of $>10 \%$, while the 99 th percentile is a 
precise concentration $(\mathrm{CV}<10 \%){ }^{7}$ HS-cTnT determinations were performed blinded to the clinical assessment of the emergency physicians.

\section{Copeptin measurement}

Copeptin was measured in heparinised blood samples collected on admission. The assay was performed on a KRYPTOR analyser using ThermoFisher Scientific sandwich immunoluminometric assay (B.R.A.H.M.S Copeptin KRYPTOR, B.R.A.H.M.S Aktiengesellschaft, Hennigsdorf, Germany). The assay principle is based on TRACE technology (Time-Resolved Amplified Cryptate Emission). The lower detection limit is $4.8 \mathrm{pmol} / \mathrm{L}$, and the functional assay sensitivity (20\% CV value) is $<12 \mathrm{pmol} / \mathrm{L}$ (data from manufacturer, recommended threshold value for this method). Copeptin determinations were performed blinded to the clinical assessment of the emergency physicians.

\section{Statistical analysis}

Continuous variables are presented as means $\pm \mathrm{SD}$, and categorical variables are expressed as numbers (percentage). Continuous variables were compared by using the Kruskal-Wallis test, and categorical variables were assessed using Pearson's $\chi^{2}$ test. Number of misclassified patients and number of correctly ruled out patients were collected for each threshold strategy, and correspond to the false negative and the true positive patients, respectively.

Receiver operating characteristic (ROC) curves were constructed to assess the sensitivity and specificity, positive predictive value and NPV throughout the concentrations of cTnI, HS-cTnT and copeptin for the diagnosis of NSTEMI, and according to the CPO. cTn and copeptin values were log-transformed before combination for ROC analysis. For cTnI values, as they were obtained from two non-standardised methods, values were normalised by factorising to the 99th percentile of the method prior to ROC analysis in order to remove any bias due to methodological differences.

Diagnostic thresholds that were used for classification of the data are:

For cTnI, the LoQvalues: $0.04 \mu \mathrm{g} / \mathrm{L}$ ( $40 \mathrm{ng} / \mathrm{L}$ ) for Bicêtre and Montpellier hospitals, $0.14 \mu \mathrm{g} / \mathrm{L}(140 \mathrm{ng} / \mathrm{L})$ for other sites.

For HS-cTnT, The LoB $(3 \mathrm{ng} / \mathrm{L})$, the LoD $(5 \mathrm{ng} / \mathrm{L})$ and the 99 th percentile $(14 \mathrm{ng} / \mathrm{L})$.

For copeptin, the manufacturer's recommended threshold at $12 \mathrm{pmol} / \mathrm{L}$.

All data are presented with their 95\% CIs. All hypothesis testing was two tailed, and $\mathrm{p}<0.05$ was considered statistically significant. Statistical analysis was performed using MedCalc (MedCalc Software, V.12.4.0.0, Mariakerke, Belgium).

\section{RESULTS \\ Characteristics of the studied population}

Table 2 shows the characteristics of the studied population. Briefly, in the total cohort mean age was $58 \pm 17$ years, and included more males. A gold-standard diagnosis of

Table 2 Main characteristics of the studied population

\begin{tabular}{|c|c|c|c|c|}
\hline & All patients & $\begin{array}{l}\text { CPO <2hours } \\
\text { (very early presenters) }\end{array}$ & CPO 2-4 hours & CPO 4-6hours \\
\hline$n$ & 449 & 160 & 143 & 146 \\
\hline Men & $281(63)$ & $101(63)$ & $96(67)$ & $84(58)$ \\
\hline \multicolumn{5}{|l|}{ Medical history } \\
\hline $\begin{array}{l}\text { Personal history of CAD } \\
\text { Dyslipidaemia } \\
\text { Diabetes } \\
\text { Smoking } \\
\text { Hypertension }\end{array}$ & $\begin{array}{l}120(27) \\
168(37) \\
67(15) \\
176(39) \\
158(35)\end{array}$ & $\begin{array}{l}38(24) \\
61(38) \\
20(13) \\
72(45) \\
52(33)\end{array}$ & $\begin{array}{l}42(29) \\
57(40) \\
22(15) \\
51(37) \\
58(41)\end{array}$ & $\begin{array}{l}40(27) \\
50(34) \\
25(17) \\
53(36) \\
48(33)\end{array}$ \\
\hline \multicolumn{5}{|l|}{ Final diagnostic } \\
\hline $\begin{array}{l}\text { NSTEMI } \\
\text { Other }\end{array}$ & $\begin{array}{l}55(12) \\
394(88)\end{array}$ & $\begin{array}{l}15(9) \\
145(91)\end{array}$ & $\begin{array}{l}22(13) \\
121(85)\end{array}$ & $\begin{array}{l}18(12) \\
128(88)\end{array}$ \\
\hline
\end{tabular}

Results are expressed in mean \pm SD or in number (percentage).

${ }^{*}$ Missing data exist for this variable.

CAD, coronary artery disease; CPO, chest pain onset; NSTEMI, non-ST-segment elevation myocardial infarction. 

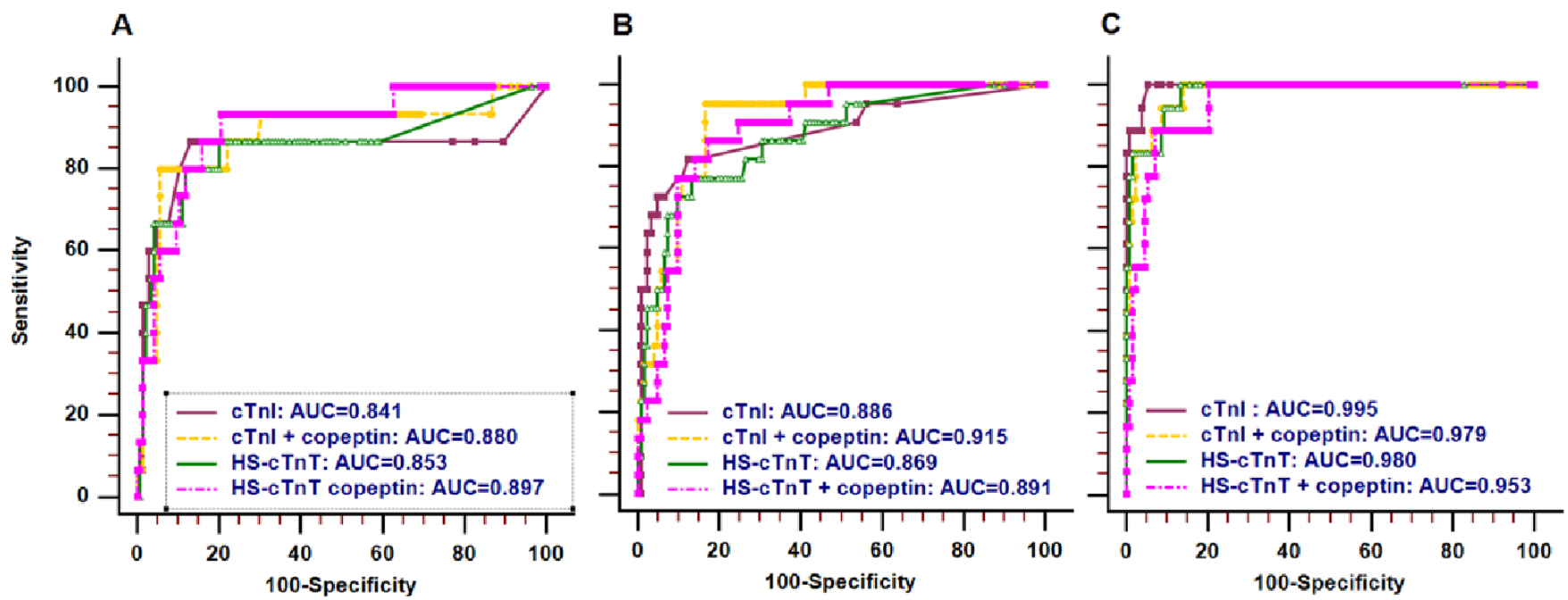

Figure 2 ROC curves of $\mathrm{cTn}$ for the diagnosis of NSTEMI, alone or in combination with copeptin. (A) CPO $<2$ hours; (B) CPO 2-4 hours; (C) CPO >4 hours. AUC, area under the ROC curve; cTn, cardiac troponin; cTnl, cardiac troponin I; CPO, chest pain onset; HS-cTnT, high-sensitivity cardiac troponin T; ROC, receiver operating characteristic; NSTEMI, non-ST-segment elevation myocardial infarction.

NSTEMI was adjudicated in 55 patients (12\%). Delay from CPO to ED admission was $<2$ hours in $160(36 \%)$ patients, was from 2 to 4 hours in $143(32 \%)$ and was $>4$ hours (and below 6 hours) in $146(32 \%)$ patients. Very early presenters with NSTEMI $(\mathrm{n}=15)$ tended to be older than those without NSTEMI $(\mathrm{n}=145)$ ( 65 vs 55 years old), were more frequently hospitalised (93\% vs $58 \%$ ), and also more frequently underwent diagnostic coronary angiography ( $73 \%$ vs $27 \%)$.

\section{Diagnostic performances according to СPO}

ROC curves of cTn for the diagnosis of NSTEMI, alone or in combination with copeptin, are presented in figure 2. Diagnostic accuracies of cTnI, cTnI +copeptinand HS-cTnT are reduced when time to CPO gets less, as indicated by estimated area under the ROC curve (AUCs) and their $95 \%$ CIs (table 3). The AUCs of HS-cTnT + copeptin were not different through the CPO categories.

Diagnostic performances of cTn, alone or in combination with copeptin, and using different decisional thresholds, are presented in table 4 .

In very early presenters ( $\mathrm{CPO}<2$ hours), a single value of cTnI alone had low sensitivity (73\% (95\% CI 73\% to $91 \%)$ ) but high specificity ( $97 \%$ ( $95 \%$ CI $92 \%$ to $99 \%)$ ), and misclassified 4 of 15 NSTEMI patients. However, this strategy could correctly rule out 141 patients (88\%). Combining copeptin with cTnI increases sensitivity, and lowers the number of misdiagnosed patients from 4 to 2 , but significantly lowers the rate of ruled out patients from $88 \%$ to $54 \%$ (table 4). Of note, addition of copeptin also significantly lowered specificity. At a threshold of $14 \mathrm{ng} / \mathrm{L}$, HS-cTnT had low sensitivity (80\% (95\% CI 59\% to $98 \%)$ ), misclassified 3 NSTEMI patients but could correctly rule out $123(79 \%)$ patients, which is less than using cTnI. The sensitivity of HS-TnT alone is likely to be suboptimal in early presenters, but can be improved by using a lower threshold for positivity or adding copeptin (but this is accompanied with a marked loss of specificity). The addition of copeptin induced a decrease in misclassified patients from 2 to 1 , either with an HS-cTnT threshold at 14 or at $5 \mathrm{ng} / \mathrm{L}$. This ultimate misdiagnosed NSTEMI patient with CPO $<2$ hours who presented with all undetectable biomarkers was a 44-year-old woman with a history of smoking and no CV risk factors; the CPO was 45 min before hospital admission (table 5 ).

\begin{tabular}{|c|c|c|c|}
\hline & Biomarker & AUC & $95 \% \mathrm{CI}$ \\
\hline \multirow{4}{*}{$\begin{array}{l}\text { CPO <2 hours } \\
\text { (very early } \\
\text { presenters) }\end{array}$} & cTnl & 0.841 & 0.775 to 0.894 \\
\hline & cTnl+copeptin & 0.880 & 0.819 to 0.926 \\
\hline & HS-cTnT & 0.853 & 0.789 to 0.904 \\
\hline & $\begin{array}{l}\text { HS- } \\
\text { cTnT+copeptin }\end{array}$ & 0.897 & 0.840 to 0.940 \\
\hline \multirow{4}{*}{$\begin{array}{l}\text { CPO } \\
2-4 \text { hours }\end{array}$} & cTnl & 0.886 & 0.823 to 0.933 \\
\hline & cTnl+copeptin & 0.915 & 0.857 to 0.955 \\
\hline & HS-cTnT & 0.869 & 0.802 to 0.919 \\
\hline & $\begin{array}{l}\text { HS- } \\
\text { cTnT+copeptin }\end{array}$ & 0.891 & 0.829 to 0.937 \\
\hline \multirow[t]{4}{*}{$\mathrm{CPO}>4$ hours } & cTnl & 0.995 & 0.965 to 1.000 \\
\hline & cTnl+copeptin & 0.979 & 0.940 to 0.995 \\
\hline & HS-cTnT & 0.980 & 0.942 to 0.996 \\
\hline & $\begin{array}{l}\text { HS- } \\
\text { cTnT+copeptin }\end{array}$ & 0.953 & 0.905 to 0.981 \\
\hline
\end{tabular}

AUC, area under the ROC curve; CPO, chest pain onset; cTnl, cardiac troponin I; HS-cTnT, high-sensitivity cardiac troponin T. 


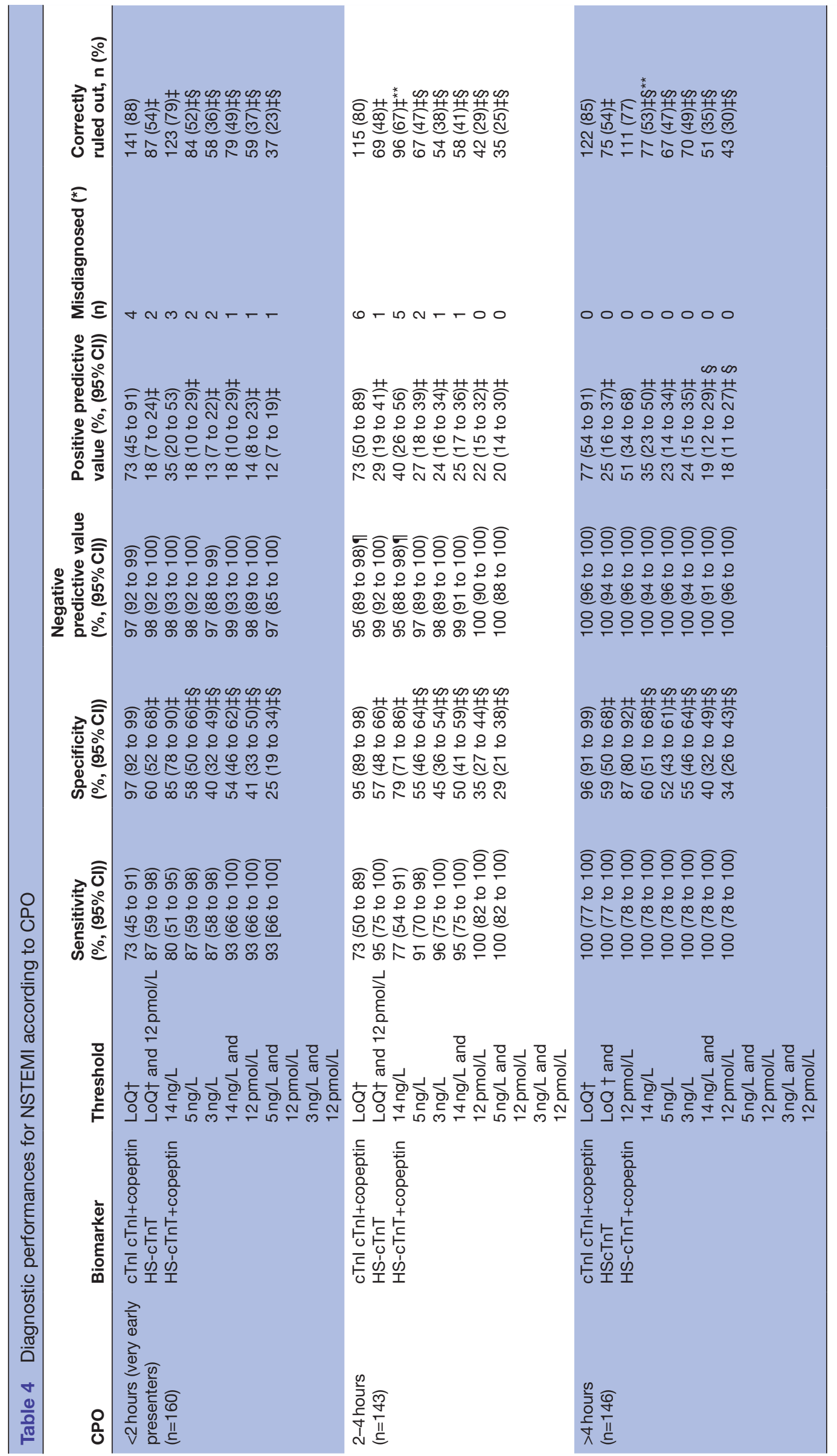




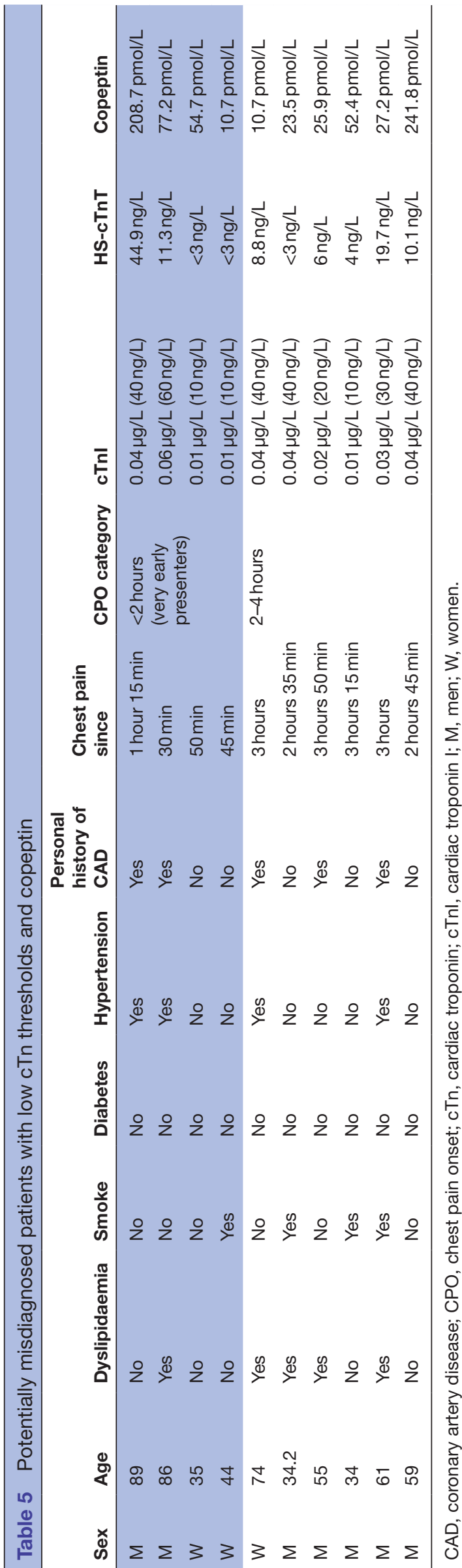

In patients with CPO 2-4hours, results are similar to those observed in very early presenters, although the number of misclassified patients was different (table 4). Adding copeptin to cTnI induced a decrease of misclassified patients from 5 to 1 for an HS-cTnT threshold at $14 \mathrm{ng} / \mathrm{L}$, and from 2 to 0 for an HS-cTnT threshold at $5 \mathrm{ng} / \mathrm{L}$. In particular, combining copeptin to the LoD $(5 \mathrm{ng} / \mathrm{L})$ of HS-cTnT reached $100 \%$ sensitivity of the test. Similar performance was observed using the LoB (3ng/L) of HS-cTnT. Indeed, all NSTEMI patients with CPO 2-4hours had a detectable HS-cTnT and/or an elevated copeptin. In this subgroup again, the use of copeptin lowered significantly the specificity of the test.

As expected, in patients with $\mathrm{CPO}>4$ hours, all patients had quantitative cTnI and detectable HS-cTnT. No patient was misclassified. The addition of copeptin in this subgroup had no effect on sensitivity or misclassified patients.

\section{Potential misdiagnosed NSTEMI}

Characteristics of potential misdiagnosed NSTEMI patients are detailed in table 5. All potentially missed NSTEMI in the very early presenters population had a $\mathrm{CPO}<1$ hour. We found no distinguishing characteristics in misclassified patients when comparing to correctly diagnosed patients in terms of age, sex and cardiovascular risks, in each CPO category. Of note, when patients with STEMI were included in our analysis, results were comparable (data not shown).

\section{DISCUSSION}

Our results indicate that diagnostic performances of HS-cTnT values at admission, alone or combined with copeptin, are reduced in the subgroup of patients with shorter CPO. Emergency physicians may not rule out NSTEMI in very early presenters with a single low value of HS-cTnT and/or copeptin at presentation.

Our studied population included one-third of early presenters, and this proportion is similar to that found by Boeddinghaus et al (26\% of patients presented within 2 hours from CPO), ${ }^{4}$ by Keller et al $(37 \%$ and $38 \%$ of patients with $\mathrm{CPO}<3$ hours, $\left.{ }^{819}\right)$ and by Reichlin et al $(222$ patients with $\mathrm{CPO}<3$ hours out of 718 , ie, $31 \%) .{ }^{20}$ More recently, Stallone et al reported 519 (26\%) patients that arrived within 2 hours of symptom onset to the $\mathrm{ED},{ }^{6}$ and Twerenbold et alreported the largest subgroup investigated so far with 1322 early presenters (with $\mathrm{CPO}<3$ hours) out of 4368 , that is, $30 \% .^{21}$ This may reflect that our study was performed in large urban areas equipped with prehospital emergency ambulances. A more prolonged delay might be expected in more rural regions. The exact definition of very early presenters is still a matter of debate. Authors nevertheless agree to define them as presenting before 2 hours. ${ }^{20}$ Very few studies examined the specific groups of early/very early presenters ${ }^{46820}$ In the ESC guidelines, a different strategy is recommended for patients with versus without $\mathrm{CPO}<6$ hours $\left(0 / 3\right.$ hours algorithm). ${ }^{1}$ Earlier 
presenters are taken into account in the 0/1-hour algorithm, but in this strategy the rapid exclusion with a unique measurement at admission (H0) is only applicable if CPO $>3$ hours. ${ }^{1}$ We believe that this proportion is not negligible and the impact of this very early population might be underestimated in studies were the CPO is not evaluated.

We observed that AUCs of cTnI, cTnI + copeptinand high-sensitivity cardiac troponin $\mathrm{T}$ (HS-cTnT) were significantly lower when CPO $<4$ hours. The NPVs were not significantly impacted by the CPO, but a suboptimal sensitivity and a non-negligible proportion of misclassified NSTEMI was observed in patients with $\mathrm{CPO}<2$ hours for all tested thresholds and combinations, even if cTnI and HS-cTnT performances can be improved by using a lower threshold for positivity or adding copeptin. The same was true for patients with CPO 2-4hours, except when using the combination of HS-cTnT $<5 \mathrm{ng} / \mathrm{L}$ and copeptin $<12$ $\mu \mathrm{mol} / \mathrm{L}$. We reported the number of misclassified patients in addition to sensitivity and NPV, because NPV is known to be dependent of the prevalence, thus its value might be biased. The absolute number of misdiagnosed patients might be more clinically pertinent than NPV. ${ }^{22}$

As previously suggested, ${ }^{1}$ we found that sensitivity and NPV of a single measurement of HS-cTn at admission seems not safe enough to exclude an NSTEMI in very early presenters. We here show that lowering HS-cTn decisional threshold to LoD or to LoB is not sufficient to detect all NSTEMI among very early chest pain presenters. Although combining copeptin to HS-cTnT increased sensitivity and lowered number of misclassified patients, none of the tested strategies allowed for identification of all NSTEMI. Results obtained in very early presenters were very similar to patients with CPO 2-4hours, except that in this later subgroup combining copeptin with HS-cTnT succeeded in significantly increasing sensitivity (all NSTEMI patients had a detectable HS-cTnT and/or an elevated copeptin).

Our results can be compared with those of Boeddinghaus $e t$ al who found that sensitivity of a single HS-cTn measurement is lower in very early presenters, in comparison to all patients. ${ }^{4}$ These authors indicated that using a single cut-off approach, $61 \%$ of the very early presenters were ruled out, which resulted in a sensitivity of $94 \%$ and an NPV of $98 \%$. They conclude that the single cut-off strategy should not be applied in early presenters. However, the authors did not evaluate the impact of copeptin across CPO categories. In another study, the same authors indicated that the additional use of copeptin did not sufficiently improve diagnostic accuracy in early presenters. ${ }^{23}$ Here again, our results are in accordance with those of Boeddinghaus et al indicating that copeptin did not improve diagnostic accuracy of hs-cTnI at presentation in early presenters. ${ }^{23}$ Mueller et al showed, using the rapid 0/1-hour algorithm that $63 \%$ patients with $\mathrm{CPO}<6$ hours were classified as rule out. However, seven patients were missed $(0.9 \%$ rate), in whom three had HS-cTnT $<$ LoD at presentation and at 1 hour. ${ }^{2}$ Moreover, a single cut-off value for HS-cTnT at $14 \mathrm{ng} / \mathrm{L}$ at presentation resulted in a sensitivity of $88.7 \%$ and an NPV of $97.3 \%$, and performed less adequately than the combination of HS-cTnT at presentation with 1-hour level and 1-hour absolute change. ${ }^{2}$ In a more recent study, Mokhtari et al evaluated a rapid 0/1-hour protocol for discharge chest pain patients based on a single value less than LoD at admission. ${ }^{24}$ These authors found two missed patients in their population, and recognise that the safety of such rapid protocol is not clear in very early presenters. They further recommend additional HS-cTnT testing at 3 hours for very early presenters. ${ }^{24}$

Performance of cTns, although measured using HS assays, might be limited in very early presenters because of their kinetics of release into the blood circulation. ${ }^{8}$ The release of cTn into the circulation following cardiomyocyte damage is a time-dependent phenomenon, ${ }^{25}$ and a single measurement approach may fail at identifying AMI very early after the onset of symptoms. ${ }^{26}$ Indeed we, like other authors, found very early presenters with undetectable HS-cTnT at admission. Time-dependent release of copeptin during AMI has been described earlier, and this biomarker has been considered as an early biomarker. ${ }^{8}$ Copeptin increases immediately after induction of ischaemia, and peaks $90 \mathrm{~min}$ after. ${ }^{27}$ However, some authors recently indicated that copeptin kinetics might be different in NSTEMI in comparison to STEMI, and that if copeptin is increased at first medical contact in the ambulance, the circulating concentrations may rapidly decrease down to normal ranges at the time of hospital admission. ${ }^{28}$ Our results are in accordance with this observation, as we found one misdiagnosed NSTEMI very early presenter with non-elevated copeptin.

Lastly, our results are reinforced by those of Stallone et al who found that the additional use of copeptin did not increase diagnostic accuracy in very early presenters. ${ }^{6}$ Furthermore, the NPV for the combination of HS-cTnT and copeptin was lower in patients arriving in the first 2 hours than in those arriving after 2 hours. ${ }^{6}$ However, these authors did not evaluate the LoD nor LoB of HS-cTnT in their work. We here report that even when lowering the cut-off of HS-cTnT, the combination of HS-cTnT and copeptin seems not enough to detect all NSTEMI among all very early presenters. Our study and the one of Stallone $e t a t^{6}$ are in accordance with previous studies that have shown that there is no or marginal benefit when adding copeptin to HS-cTn assays; indeed, Wildi $e$ al indicated that copeptin provides no significant increase in AUC when combined to HS-cTn, ${ }^{29}$ either in their all population or in patients with $\mathrm{CPO}<4$ hours. These authors found an incremental value in sensitivities, NPV and calculating the integrated discrimination improvement index, but they did not evaluate low HS-cTn thresholds such as $\mathrm{LoB}$ and $\mathrm{LoD}$ values. ${ }^{29}$

Of note, all patients with CPO $>4$ hours had detectable cTnI and HS-cTnT, that is, the use of copeptin in this situation added no gain. Other studies reported that copeptin testing for the rule out of NSTEMI should be 
limited to $\mathrm{CPO}<6$ hours. ${ }^{13}$ According to our data, the added value of copeptin might be more restricted, but further studies are needed to confirm our findings.

The current ESC guidelines incorporate an additional criterion for direct rule out of patients that are not very early presenters; indeed, the rapid rule out using a single measurement at admission is possible only if CPO is $>3$ hours. ${ }^{1}$ Furthermore, this rapid algorithm can be used only for 3 HS-cTn assays, including HS-cTnT. Considering our data about patients with CPO $>4$ hours, we note that our conclusions are in line with the recommendations. Therefore, the use of a single measurement at admission might be used for a safe rule out in patients that are not very early presenters. The alternative rule out criteria, combining baseline concentration and 1-hour change, should be used in early presenters.

\section{Limitations of our study}

First, it is a post hoc analysis of three previously published studies, and some data are missing (vital signs at admission, details in ECG findings, eg). Second, only a single measurement of troponin at admission was considered for this analysis, and we did not evaluate its kinetics; we, therefore, cannot comment on the accuracy of the recent 1-hour algorithm in our population. ${ }^{12}$ Third, different cTn assays were used across the different centres ${ }^{11-13}$ and we had to normalise cTnI values before analysis in order to minimise bias. However, all centres evaluated the same HS-cTnT and copeptin. Fourth, as the gold-standard diagnosis was based on a non-HS cTn, we recognise that this could result in underdiagnosis of myocardial injury. Previous studies have shown that an early rule out of NSTEMI using Hs-cTn alone, also in the vulnerable subgroup of early presenters, is safe (with high AUC, sensitivities and NPV in patients presenting with $\mathrm{CPO}<3$ hours) ${ }^{20}$ In our study, the combined biomarkers are not safe enough for early rule out of NSTEMI in patients presenting very early ( $\mathrm{CPO}<2$ hours). The fact that gold-standard diagnoses were adjudicated by the use of a conventional but not a Hs-cTn assay, and that different assays where used, may have led to underdiagnose patients. This point limits the generalisability of our findings and explains why sensitivities and NPVs were much lower as compared with previous studies. However, our results are comparable to those of Stallone et al how recently used an HS-cTn, as suggested by our AUC (0.85 (95\% CI 0.79 to 0.90$)$ ) in comparison to those of Stallone (0.86 (95\% CI 0.82 to 0.090$)) .{ }^{6}$ However, the aim of our work is not to evaluate another time global HS-cTn accuracy, but to highlight CPO effects on diagnostic accuracy of HS-cTn combined or not to copeptin. Fifth, we examined three subgroups with CPO $<2$ hours, $2-4$ hours and 4 hours and defined very early presenters as those having $\mathrm{CPO}<2$ hours, as based on the accepted definition of early presenters. ${ }^{20}$ Even if very early presenters represent more than one-third of the studied population, the number of very early presenters (CPO $<2$ hours) is relatively small, although data from three cohorts were used. This explains why false rule out of three patients results in a significant drop in sensitivity and NPV. Many previous studies investigated the rule out performance in early presenters using, for example, the LoD of Hs-cTnT and Hs-cTnI and found much higher sensitivities and NPVs. This can be explained due to a larger number of patients. ${ }^{420}$

\section{CONCLUSION}

A single measurement of HS-cTnT alone or in combination with copeptin at admission seems not sensitive enough to safely rule out NSTEMI in very early presenters (CPO <2 hours from ED admission). If other studies confirm our findings, another strategy to safely exclude NSTEMI in this specific population that represents one-third of patients with chest pain is warranted.

\section{Author affiliations}

${ }^{1}$ Service de Diagnostic Biologique Automatisé, Hôpital Cochin, Hôpitaux Universitaires Paris Centre (HUPC), Assistance Publique des Hôpitaux de Paris (APHP), Paris, France

²Département des Urgences, Hôpital Lapeyronie, CHU de Montpellier, Montpellier, France

${ }^{3}$ Service de Cardiologie, Hôpital Avicenne, Hôpitaux Universitaires Paris Seine Saint Denis, Assistance Publique des Hôpitaux de Paris (AP-HP), Bobigny; Université Paris, Paris, France

${ }^{4}$ INSERM UMR S-942, INSERM, Paris, France

${ }^{5}$ Département de Biochimie, Hôpital Lapeyronie - CHU Montpellier, Montpellier, France

${ }^{6}$ Laboratoire de Biochimie et Hormonologie, Hôpital Tenon, Hôpitaux Universitaires

Est Parisien, Assistance Publique des Hôpitaux de Paris (AP-HP), Paris, France

${ }^{7}$ Sorbonne Universités UPMC-University Paris, Biosfast, Paris

${ }^{8}$ Urgences-SMUR, Centre Hospitalier Eure-Seine - Hôpital d'Evreux, Rue Léon Schwartzenberg, Évreux, France

${ }^{9}$ Centre Régional Universitaire des Urgences, Hôpital François Mitterrand, 5

Boulevard Jeanne d'Arc, Dijon, France

${ }^{10}$ Université de Bourgogne, Dijon, France

Contributors PR, CM, MS and NP contributed to the design and inclusions. CC-G, SL, A-MD and GL realised sample collections and biomarker measurements. CC-G and PR realised statistical analysis. All authors approved the final manuscript. ThermoFisher Scientific and Roche Diagnostic provided reagents.

Funding The authors have not declared a specific grant for this research from any funding agency in the public, commercial or not-for-profit sectors.

Competing interests CC-G, PR and CM received honorarias and lecture fees from Roche Diagnostics andThermofisher Scientific.

Patient consent for publication Not required.

Ethics approval All studies received approval from local institutional review board. The study complied with the principles of the Declaration of Helsinki.

Provenance and peer review Not commissioned; externally peer reviewed.

Data sharing statement Extra data can be accessed via the Dryad data repository at with the doi: https://doi.org/10.5061/dryad.8t87571.

Open access This is an open access article distributed in accordance with the Creative Commons Attribution Non Commercial (CC BY-NC 4.0) license, which permits others to distribute, remix, adapt, build upon this work non-commercially, and license their derivative works on different terms, provided the original work is properly cited, appropriate credit is given, any changes made indicated, and the use is non-commercial. See: http://creativecommons.org/licenses/by-nc/4.0/.

\section{REFERENCES}

1. Roffi M, Patrono C, Collet JP, et al. 2015 ESC Guidelines for the management of acute coronary syndromes in patients presenting without persistent ST-segment elevation: Task Force for the Management of Acute Coronary Syndromes in Patients Presenting 
without Persistent ST-Segment Elevation of the European Society of Cardiology (ESC). Eur Heart J 20162016;37:267-315.

2. Mueller C, Giannitsis E, Christ M, et al. Multicenter Evaluation of a 0-Hour/1-Hour Algorithm in the Diagnosis of Myocardial Infarction With High-Sensitivity Cardiac Troponin T. Ann Emerg Med 2016;68:76-87.

3. Wildi K, Nelles B, Twerenbold R, et al. Safety and efficacy of the 0 $\mathrm{h} / 3 \mathrm{~h}$ protocol for rapid rule out of myocardial infarction. Am Heart $\mathrm{J}$ 2016;181:16-25.

4. Boeddinghaus J, Nestelberger T, Twerenbold R, et al. Direct Comparison of 4 Very Early Rule-Out Strategies for Acute Myocardial Infarction Using High-Sensitivity Cardiac Troponin I. Circulation 2017;135:1597-611.

5. Pickering JW, Than MP, Cullen L, et al. Rapid Rule-out of Acute Myocardial Infarction With a Single High-Sensitivity Cardiac Troponin T Measurement Below the Limit of Detection: A Collaborative Metaanalysis. Ann Intern Med 2017;166:715-24.

6. Stallone F, Schoenenberger AW, Puelacher C, et al. Incremental value of copeptin in suspected acute myocardial infarction very early after symptom onset. Eur Heart J Acute Cardiovasc Care 2016;5:407-15.

7. Chenevier-Gobeaux C, Lefevre G, Bonnefoy-Cudraz E, et al. Why a new algorithm using high-sensitivity cardiac troponins for the rapid rule-out of NSTEMI is not adapted to routine practice. Clin Chem Lab Med 2016;54:e279-80.

8. Keller T, Tzikas S, Zeller T, et al. Copeptin improves early diagnosis of acute myocardial infarction. J Am Coll Cardiol 2010;55:2096-106.

9. Reichlin T, Hochholzer W, Stelzig C, et al. Incremental value of copeptin for rapid rule out of acute myocardial infarction. J Am Coll Cardiol 2009;54:60-8.

10. Lipinski MJ, Escárcega RO, D'Ascenzo F, et al. A systematic review and collaborative meta-analysis to determine the incremental value of copeptin for rapid rule-out of acute myocardial infarction. Am J Cardiol 2014;113:1581-91.

11. Raskovalova T, Twerenbold R, Collinson PO, et al. Diagnostic accuracy of combined cardiac troponin and copeptin assessment for early rule-out of myocardial infarction: a systematic review and metaanalysis. Eur Heart J Acute Cardiovasc Care 2014;3:18-27.

12. Chenevier-Gobeaux $C$, Freund $Y$, Claessens YE, et al. Copeptin for rapid rule out of acute myocardial infarction in emergency department. Int J Cardiol 2013;166:198-204.

13. Möckel M, Searle J, Hamm C, et al. Early discharge using single cardiac troponin and copeptin testing in patients with suspected acute coronary syndrome (ACS): a randomized, controlled clinical process study. Eur Heart $J$ 2015;36:369-76.

14. Sebbane M, Lefebvre S, Kuster N, et al. Early rule out of acute myocardial infarction in ED patients: value of combined highsensitivity cardiac troponin T and ultrasensitive copeptin assays at admission. Am J Emerg Med 2013;31:1302-8.
15. Chenevier-Gobeaux C, Meune C, Lefevre G, et al. A single value of high-sensitive troponin $\mathrm{T}$ below the limit of detection is not enough for ruling out non ST elevation myocardial infarction in the emergency department. Clin Biochem 2016;49:1113-7.

16. Bossuyt PM, Reitsma JB, Bruns DE, et al. The STARD statement for reporting studies of diagnostic accuracy: explanation and elaboration. Ann Intern Med 2003;138:W1-12.

17. Hamm CW, Bassand JP, Agewall S, et al. ESC Guidelines for the management of acute coronary syndromes in patients presenting without persistent ST-segment elevation: The Task Force for the management of acute coronary syndromes (ACS) in patients presenting without persistent ST-segment elevation of the European Society of Cardiology (ESC). Eur Heart J 2011;32:2999-3054.

18. Thygesen K, Alpert JS, Jaffe AS, et al. Third universal definition of myocardial infarction. Circulation 2012:126:2020-35.

19. Keller T, Zeller T, Ojeda F, et al. Serial changes in highly sensitive troponin I assay and early diagnosis of myocardial infarction. JAMA 2011;306:2684-93.

20. Reichlin T, Hochholzer W, Bassetti S, et al. Early diagnosis of myocardial infarction with sensitive cardiac troponin assays. $N$ Engl $J$ Med 2009;361:858-67.

21. Twerenbold R, Neumann JT, Sörensen NA, et al. Prospective Validation of the 0/1-h Algorithm for Early Diagnosis of Myocardial Infarction. J Am Coll Cardiol 2018;72:620-32.

22. Righini M, Aujesky D, Roy PM, et al. Clinical usefulness of D-dimer depending on clinical probability and cutoff value in outpatients with suspected pulmonary embolism. Arch Intern Med 2004;164:2483-7.

23. Boeddinghaus $\mathrm{J}$, Reichlin $\mathrm{T}$, Nestelberger $\mathrm{T}$, et al. Early diagnosis of acute myocardial infarction in patients with mild elevations of cardiac troponin. Clin Res Cardiol 2017;106:457-67.

24. Mokhtari A, Lindahl B, Schiopu A, et al. A 0-Hour/1-Hour Protocol for Safe, Early Discharge of Chest Pain Patients. Acad Emerg Med 2017;24:983-92.

25. White HD. Pathobiology of troponin elevations: do elevations occur with myocardial ischemia as well as necrosis? J Am Coll Cardiol 2011;57:2406-8.

26. Korley FK. The Wait for High-Sensitivity Troponin Is Over-Proceed Cautiously. JAMA Cardiol 2018;3:112-3.

27. Gu YL, Voors AA, Zijlstra F, et al. Comparison of the temporal release pattern of copeptin with conventional biomarkers in acute myocardial infarction. Clin Res Cardiol 2011;100:1069-76.

28. Slagman A, Searle J, Müller C, et al. Temporal release pattern of copeptin and troponin $\mathrm{T}$ in patients with suspected acute coronary syndrome and spontaneous acute myocardial infarction. Clin Chem 2015;61:1273-82.

29. Wildi K, Zellweger C, Twerenbold R, et al. Incremental value of copeptin to highly sensitive cardiac Troponin I for rapid rule-out of myocardial infarction. Int J Cardiol 2015;190:170-6. 